\title{
Challenging Management of Neovascular Glaucoma to Achieve the Best Visual Outcome
}

\author{
Ikhwanuliman Putera Astrianda N. Suryono Widya Artini \\ Department of Ophthalmology, Faculty of Medicine, University of Indonesia - Cipto \\ Mangunkusumo Kirana Hospital, Jakarta, Indonesia
}

\section{Keywords}

Ahmed valve - Anti-vascular endothelial growth factor - Diabetic retinopathy - Neovascular glaucoma Panretinal photocoagulation

\begin{abstract}
Neovascular glaucoma (NVG) resulting from ischemic insults caused by various diseases, such as proliferative diabetic retinopathy and central vein occlusion, remains a challenging situation. This case report aims to describe the complex management of NVG, resulting from diabetic retinopathy, to achieve the best visual outcome. A 47-year-old male presented with poor visual acuity of both eyes. His right eye was inoperable. Ahmed valve implantation with intraoperative intravitreal bevacizumab injection was performed in his left eye. Panretinal photocoagulation was performed serially after phacoemulsification. However, intraocular pressure was found to be raised, thus necessitating 5 -fluorouracil needling bleb revision and administration of intracameral bevacizumab injection. During 15 months of follow-up we were able to achieve the best visual outcome possible in this patient. Comprehensive management of NVG should be implemented with a multidisciplinary approach.

(C) 2020 The Author(s)

Published by S. Karger AG, Basel
\end{abstract}




\section{Introduction}

Neovascular glaucoma (NVG) is a secondary glaucoma with an estimated incidence of around 6.6/10,000. Proliferative diabetic retinopathy (PDR) was reported to be the leading cause of NVG $(48.7 \%)[1,2]$. NVG is a complex symptom to treat due to its refractory nature resulting in uncontrolled intraocular pressure (IOP). The reported failure rate of medical and surgical intervention of NVG patients is $62.8 \%$, with the majority of patients suffering from total blindness [2].

The management of NVG caused by PDR needs careful and comprehensive work, not only by glaucoma/vitreoretinal specialists but also by internists/endocrinologists, as it is imperative that the blood glucose level be constantly well controlled. Management of NVG includes the administration of panretinal photocoagulation (PRP), combination antiglaucoma drugs, surgical intervention, and anti-vascular endothelial growth factor (VEGF) injection [3]. However, not all NVG eyes brought about by PDR can be directly treated with PRP, and the surgical success rate among NVG patients is still rated as significantly low compared to other types of glaucoma [3].

This case report aimed to demonstrate the complex management of NVG and shows that with comprehensive and timely treatment, it is indeed possible to achieve a good outcome in preserving visual function.

\section{Case Presentation}

A 47-year-old male presented with the complaint of his right eye having gradually become blurred over the past 4 months followed by blurred vision in his left eye 1 week before the initial visit. He reported a 10-year history of diabetes mellitus treated with metformin $3 \times$ $500 \mathrm{mg}$ and a blood glucose level controlled at around 100-200 mg/dL. On ophthalmological examination, his visual acuity showed light perception in the right eye and 20/400 in the left eye. IOP was high in both eyes (right eye $57 \mathrm{~mm} \mathrm{Hg}$ and left eye $50 \mathrm{~mm} \mathrm{Hg}$ ) with mid-dilated pupil, corneal edema, and a bullous appearance on the right eye. Rubeosis iridis was also prominent in both eyes. Funduscopic examination of the left eye showed a slightly enlarged cup-disk ratio (0.4-0.5) with neovascularization on the disk, dot blot hemorrhage, and exudates in all quadrants of the retina. Gonioscopic findings in the right eye showed closed angle with neovascularization of the angle in all quadrants, while the angles of the left eye were still open with neovascularization of the angle in three quadrants. The glucose level was found to be $300 \mathrm{mg} / \mathrm{mL}$. The patient was diagnosed as having NVG in both eyes as a result of PDR and uncontrolled diabetic mellitus. He was admitted for Ahmed valve implantation with intraoperative intravitreal $1.25 \mathrm{mg} / 50 \mu \mathrm{L}$ bevacizumab injection in the left eye. Surgical intervention was required for the severe condition of the right eye. Timolol maleate and brinzolamide eye drops together with oral carbonic anhydrase were prescribed. The patient was also referred for consultation with an endocrine specialist.

One day following Ahmed valve implantation, the IOP of the left eye was found to be reduced to $6 \mathrm{~mm} \mathrm{Hg}$, and marked improvement of visual acuity (20/100) with absence of hyphema was observed. PRP was initially planned for the patient but could not be performed due to poor visualization caused by lens opacity. Phacoemulsification with intraocular lens insertion was then performed and immediately followed by PRP. BCVA improved to 20/20. One month after PRP, the patient's IOP had gradually increased to $37 \mathrm{~mm} \mathrm{Hg}$ with flat Ahmed implant bleb and persistent rubeosis iridis, thus necessitating a 5-fluorouracil needling pro- 


\section{Case Reports in Ophthalmology}

cedure combined with bevacizumab intracameral injection. The IOP gradually decreased to $<20 \mathrm{~mm} \mathrm{Hg}$ with administration of antiglaucoma eye drops (brinzolamide and timolol maleate). The second PRP was performed in the following month. The patient's left eye BCVA was consistent at 20/20 and IOP was $9 \mathrm{~mm} \mathrm{Hg}$ at 15 months follow-up after the second PRP. He was instructed to have bi- or trimonthly visits as rubeosis iridis persisted; however, funduscopic appearance was good and further careful monitoring was advised. The patient's blood glucose level was maintained at $<200 \mathrm{~mm} / \mathrm{dL}$.

The last clinical appearance is shown in Figure 1 and Figure 2. Despite persisting rubeosis, the patient presented with consistent BCVA at 20/20 and controlled IOP. Optical coherence tomography of the optic disk (Fig. 3, left) showed good retinal nerve fiber layer thickness. Optical coherence tomography of the macula (Fig. 3, right) showed slight macular edema. Visual field examination with Humphrey test showed mild to moderate peripheral visual field defect and preserved central visual field in the left eye.

\section{Discussion}

NVG remains a challenging situation resulting from ischemic insults caused by various diseases, including PDR and central vein occlusion, and in many cases IOP may be raised due to neovascularization in the iris and angle. Ocular treatment modalities in NVG should be employed immediately to include oral and topical IOP-lowering agents, PRP, anti-VEGF, and surgical intervention. Retinal laser coagulation is a key strategy to halt NVG progression. However, PRP alone may be insufficient to prevent disease progression in severe and rapidly progressing NVG since it takes time before optimal results are seen. In this case, intraocular antiVEGF injection as an adjuvant should be administered. In many cases, intravitreal anti-VEGF injection preceded surgical intervention to regress neovascularization [4].

This case report documents a patient with rapidly progressive NVG as a result of PDR. Over a period of some months his right eye lost visual acuity and his left eye also gradually became blurred. The treatment goal for the left eye was to preserve vision by lowering IOP, discontinuing the progression of NVG, and offering definitive treatment for PDR. Despite maximum medical intervention the IOP was still uncontrolled and the patient had to undergo surgical management. Ahmed valve implantation was scheduled as the optimal surgical choice for NVG, with a slightly higher success rate than trabeculectomy [5]. Due to the progression and adverse condition in the one eye, preoperative anti-VEGF injection was not administered. Instead, intravitreal injection of bevacizumab, as one of the anti-VEGF agents, was conducted intraoperatively combined with glaucoma drainage device. One previous study suggested performing intraocular anti-VEGF preoperatively serially to halt NVG progression and to diminish neovascularization for a short period. Preoperative administration enabled prevention of intraoperative bleeding during glaucoma intervention [6]. More than $90 \%$ of intravitreally injected bevacizumab enters the systemic circulation through the retina and choroid, whereas $<5 \%$ enters through the anterior chamber [7].

VEGF is excreted by Muller cells in compromised retinal capillary status and diffuse anteriorly. Tissue damage and ischemic state stimulate pericytes and endothelial cells to shift towards the activated state. Angiogenesis steps occurred, starting with increased capillary permeability, activation, migration of endothelial cells and pericytes, and the formation of vascular sprouts. Finally, maturation of blood vessels and neovascularization occurred in the retina as well as in the anterior chamber [8]. In PDR, high VEGF indicates poor prognosis and demonstrates a more progressive disease. 
In patients with NVG as a result of PDR, Kuzmin et al. [9] showed that the VEGF level in aqueous humor was significantly higher than in the control group. The level of aqueous VEGF determines the failure of Ahmed valve implantation among patients with NVG brought about by PDR. Lim et al. [10] found that intracameral bevacizumab reduced the VEGF level in aqueous humor up to 30 times among NVG patients 2 weeks following injection. In the past, there was some concern regarding the possibility of corneal toxicity due to the intracameral route of anti-VEGF injection. This study showed that the oxidative stress parameter in the anterior chamber was raised following intracameral bevacizumab administration. However, no endothelial cell loss or dysfunction could be demonstrated [11].

Because of the temporary nature of anti-VEGF, PRP is considered the preferred treatment and should be carried out immediately. If necessary, PRP can also be done serially to prevent further disease progression. This procedure will reduce hypoxia and recover the equilibrium between proangiogenic/antiangiogenic factors in the adjacent retina. In our case, after the second PRP, the patient was instructed to have bi- or trimonthly visits with regular glucose level examinations.

Glaucoma drainage devices are usually considered the preferred treatment option for refractory glaucoma. Valve implant has gained popularity due to the reduced rate of hypotony. Shen et al. [5] found that both trabeculectomy and glaucoma drainage surgery result in a similar beneficial outcome in NVG patients. However, in another report, trabeculectomy with antimetabolite agent was reported to have successfully lowered IOP to approximately $62 \%$ after 1 year, while Ahmed implant can attain a 75\% success rate after 1 year. Other studies have reported that most traditional glaucoma surgical interventions do not effectively decrease IOP due to extensive neovascularization in the angle [12]. This percentage can be increased with combining injection of an anti-VEGF agent and glaucoma drainage device [13].

Increase of IOP after Ahmed valve implantation may be caused by hypertensive phase or early failed bleb. The hypertensive phase tends to occur, on average, 1 month and to stabilize 3-6 months after implantation of the glaucoma drainage valve [14]. Fibrosis and encapsulation of the Ahmed valve may happen in the postoperative period and obstruct the outflow of the implant. Needling procedure with antimetabolite can be performed to overcome this condition and inhibit fibrosis. Quaranta et al. [15] evaluated 5-fluorouracil needling revision of Ahmed valve bleb and found that $76 \%$ of their patients had well-controlled IOP at 24 months of follow-up. Combination of immediate PRP and intraocular anti-VEGF injection, as well as glaucoma implant, showed a good long-term outcome with close monitoring and, as a result, preserved the best visual outcome in this patient.

\section{Conclusion}

Comprehensive management should be implemented in NVG cases. Besides the administration of glaucoma and retinal care, blood glucose level should be carefully controlled during the follow-up period. Ahmed valve implantation, anti-VEGF injection (intravitreally and intracamerally), as well as PRP offer an effective method to halt NVG progression. If required, 5 -fluorouracil needling may be performed to revise the failing bleb. 


\section{Statement of Ethics}

This case report was conducted according to optimal clinical practice. The authors state that they have full control over all primary data and have no ethical conflicts to disclose.

\section{Disclosure Statement}

The authors declare no conflict of interest.

\section{Funding Sources}

The authors have no financial disclosure in any materials discussed in this report.

\section{Author Contributions}

I. Putera analyzed the patient data and was a major contributor in writing the manuscript. A.N. Suryono and W. Artini investigated and supervised the findings of this work. All authors discussed the results and contributed to the final manuscript.

\section{References}

1 Al-Bahlal A, Khandekar R, Al Rubaie K, Alzahim T, Edward DP, Kozak I. Changing epidemiology of neovascular glaucoma from 2002 to 2012 at King Khaled Eye Specialist Hospital, Saudi Arabia. Indian J Ophthalmol. 2017 Oct;65(10):969-73.

2 Noor N, Mustafa S. Karakteristik klinis dan hasil tatalaksana glaukoma neovaskular di Rumah Sakit Cipto Mangunkusumo Kirana periode 2012-2014. Jakarta: FKUI-RSCM Kirana; 2015.

3 Rodrigues GB, Abe RY, Zangalli C, Sodre SL, Donini FA, Costa DC, et al. Neovascular glaucoma: a review. Int J Retina Vitreous. 2016 Nov;2(1):26.

4 Sun Y, Liang Y, Zhou P, Wu H, Hou X, Ren Z, et al. Anti-VEGF treatment is the key strategy for neovascular glaucoma management in the short term. BMC Ophthalmol. 2016 Aug;16(1):150.

5 Shen CC, Salim S, Du H, Netland PA. Trabeculectomy versus Ahmed Glaucoma Valve implantation in neovascular glaucoma. Clin Ophthalmol. 2011;5(5):281-6.

6 Sevim MS, Buttanri IB, Kugu S, Serin D, Sevim S. Effect of intravitreal bevacizumab injection before Ahmed glaucoma valve implantation in neovascular glaucoma. Ophthalmologica. 2013;229(2):94-100.

7 Gal-Or O, Dotan A, Dachbash M, Tal K, Nisgav Y, Weinberger D, et al. Bevacizumab clearance through the iridocorneal angle following intravitreal injection in a rat model. Exp Eye Res. 2016 Apr;145:412-6.

8 Witmer AN, van Blijswijk BC, van Noorden CJ, Vrensen GF, Schlingemann RO. In vivo angiogenic phenotype of endothelial cells and pericytes induced by vascular endothelial growth factor-A. J Histochem Cytochem. 2004 Jan;52(1):39-52.

9 Kuzmin A, Lipatov D, Chistyakov T, Smirnova O, Arbuzova M, Ilin A, et al. Vascular endothelial growth factor in anterior chamber liquid patients with diabetic retinopathy, cataract and neovascular glaucoma. Ophthalmol Ther. 2013 Jun;2(1):41-51.

10 Lim TH, Bae SH, Cho YJ, Lee JH, Kim HK, Sohn YH. Concentration of vascular endothelial growth factor after intracameral bevacizumab injection in eyes with neovascular glaucoma. Korean J Ophthalmol. 2009 Sep; 23(3):188-92.

11 Akal A, Ulas T, Goncu T, Guldur ME, Kocarslan S, Taskin A, et al. Evaluating the safety of intracameral bevacizumab application using oxidative stress and apoptotic parameters in corneal tissue. Int J Ophthalmol. 2015 Aug;8(4):697-702.

12 He Y, Tian Y, Song W, Su T, Jiang H, Xia X. Clinical efficacy analysis of Ahmed glaucoma valve implantation in neovascular glaucoma and influencing factors: a STROBE-compliant article. Medicine (Baltimore). 2017 Oct; 96(42):e8350. 


\section{Case Reports in Ophthalmology}

\begin{tabular}{l|l}
\hline DOI: 10.1159/000506041 & $\begin{array}{l}\text { () 2020 The Author(s). Published by S. Karger AG, Basel } \\
\text { www.karger.com/cop }\end{array}$ \\
\hline
\end{tabular}

Putera et al:: Management of Neovascular Glaucoma

13 Sharma P, Agarwal N, Choudhry RM. Neovascular Glaucoma - A Review. Del J Ophthalmol. 2016;26(3): $170-5$.

14 Riva I, Roberti G, Oddone F, Konstas AG, Quaranta L. Ahmed glaucoma valve implant: surgical technique and complications. Clin Ophthalmol. 2017 Feb;11:357-67.

15 Quaranta L, Floriani I, Hollander L, Poli D, Katsanos A, Konstas AG. Needle Revision With 5 -fluorouracil for the Treatment of Ahmed Glaucoma Valve Filtering Blebs: 5-Fluorouracil Needling Revision can be a Useful and Safe Tool in the Management of Failing Ahmed Glaucoma Valve Filtering Blebs. J Glaucoma. 2016 Apr; 25(4):e367-71.
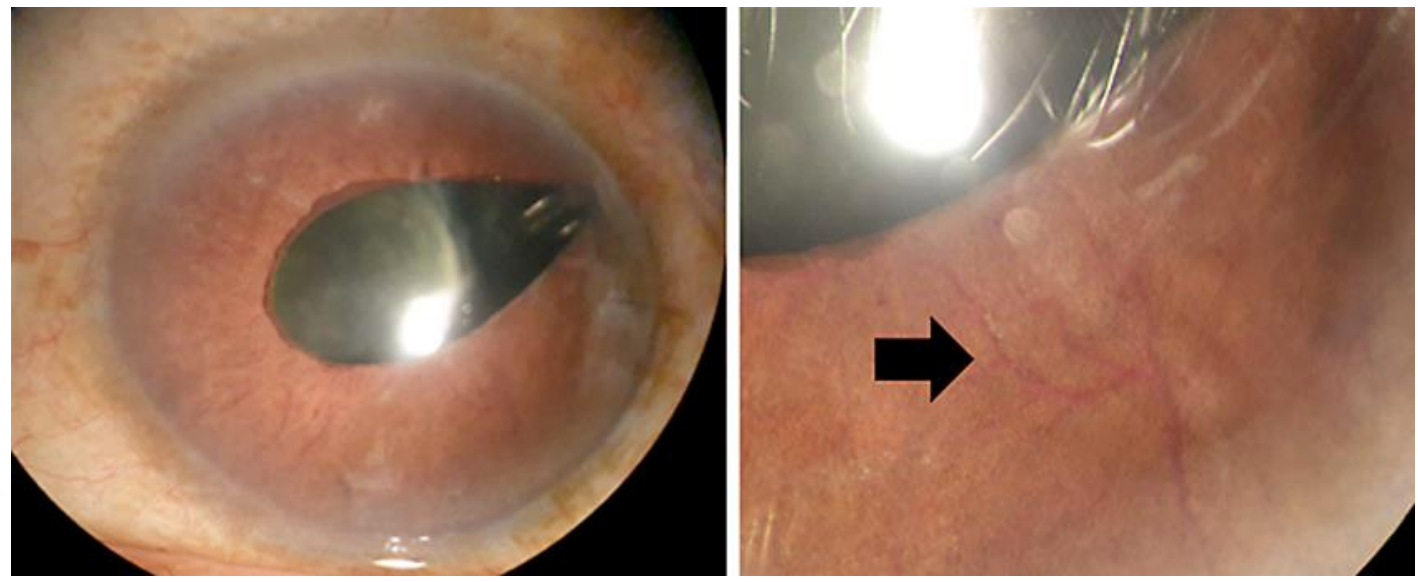

Fig. 1. Clinical appearance of the patient's left eye at 15-month follow-up showing persistent iris neovascularization (arrow).

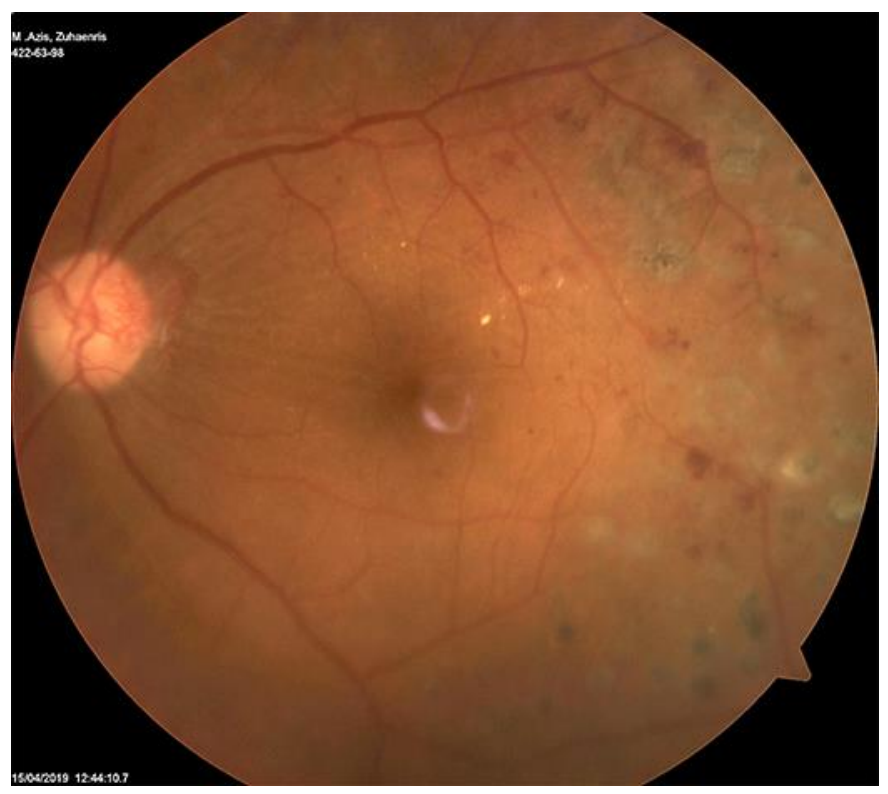

Fig. 2. Funduscopic appearance at 15-month follow-up showed relatively good retinal condition. 


\section{Case Reports in Ophthalmology}
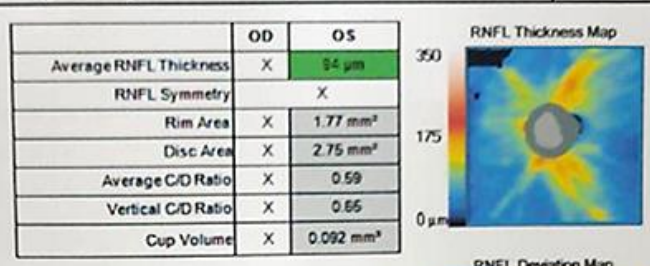

Neuro-retinal Rim Thickness
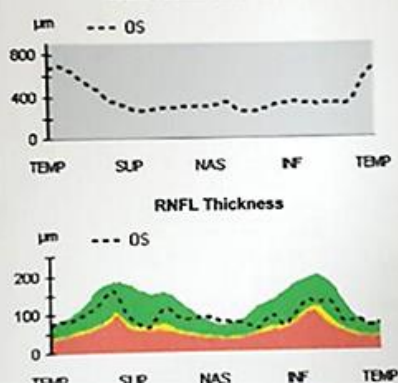
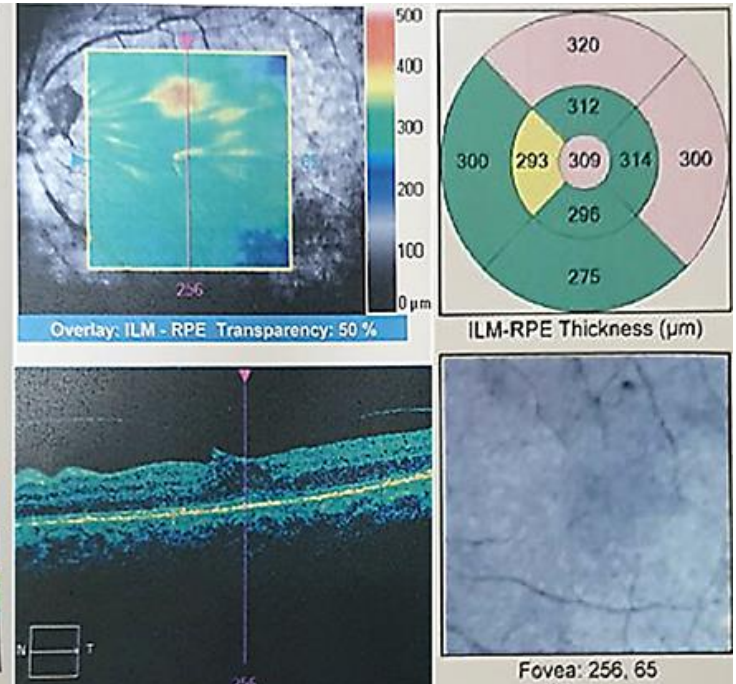

Fig. 3. Optical coherence tomography of the patient's disk (left) and macula (right). 